\title{
Photoacoustic Imaging: An Emerging Optical Modality in Diagnostic and Theranostic Medicine
}

\author{
Yao Sun ${ }^{2 *}$, Huabei Jiang ${ }^{1}$ and Brian E. O'Neill² \\ ${ }^{1}$ Department of Biomedical Engineering, University of Florida, Gainesville, FL 32611 \\ ${ }^{2}$ Department of Radiology, Translational Imaging, Methodist Hospital Research Institute, Weill Medical College, Cornell University, Houston, TX 77030
}

\begin{abstract}
Photoacoustic imaging, also referred to optoacoustic imaging, is an emerging optical imaging technique that is capable of providing both structural and functional optical information of tissues up to several centimeters deep with scalable ultrasound resolution. The combination of ultrasound spatial resolution and intrinsic rich optical contrast in cancer / inflammatory tissues allow photoacoustic imaging a promising early detection tool in diagnostic medicine. At the same time, the availability of various optical absorbing nanostructures extensively studied in targeted drug delivery extends the capability of photoacoustic imaging to theranostic medicine, where functionalized nanostructures provide photoacoustic imaging contrast and targeting moieties / drug carrier for targeted drug delivery. In this paper we provide a review of the photoacoustic imaging technique for studies in diagnostic and theranostic medicine. The technical consideration for photoacoustic imaging systems and reconstruction algorithms of structural / quantitative photoacoustic imaging have been introduced. A review of the photoacoustic imaging in breast cancer detection and osteoarthritis evaluation is included in the section of photoacoustic applications in diagnostic medicine, where patient subjects have been already recruited and photoacoustically evaluated. At the end of this paper, a brief review of optical controlled drug release and photoacoustic evaluation is presented as a view to the effort of combining laser induced photoacoustic imaging with laser controlled drug delivery / release in theranostic medicine.
\end{abstract}

\section{Introduction}

Photoacoustic imaging, also referred to optoacoustic imaging, is a hybridized imaging technique to visualize rich optical contrast in biological tissues with scalable ultrasonic resolution, by detecting outgoing broadband ultrasound signals generated from laser illuminated biological tissues [1-3]. Unlike ionizing radiation based modalities such as plain radiography, computed tomography (CT) and positron emission tomography (PET), only low energy photon and ultrasound wave are involved in photoacoustic imaging. For example, the photon energy of visible/near infrared light for photoacoustic imaging is only about $2 \mathrm{eV}$ while the energy of typical $\mathrm{x}$-ray for radiography is about $10-100 \mathrm{keV}$. As such, photoacoustic imaging is a safe optical imaging approach, particularly promising for frequent-use situation such as in routine examination or preventive examination.

Pure optical imaging methods, such as optical coherent tomography (OCT), fluorescent imaging and different kinds of optical microscopy, have been extensively used or studied in biomedicine to image cellular and biological structures or activities. These optical imaging methods take the merits of spectroscopic feature of light and tissue-light interaction, the intrinsic optical contrasts (scattering, absorption, refractive index, polarization, etc) rich in biological tissues, the availability of exogenous agents and biological techniques for contrast enhancement, cells labeling/targeting, molecular imaging and gene expression. Within the first millimeter depth, pure optical imaging methods utilize the short wavelength of coherence light and provide highly resolved images for biomedical studies in both cellular and biological scale. However, beyond millimeter depth, which is one photonic transport mean free path, photons in biological tissues are strongly scattered, restraining the spatial resolution of pure optical imaging methods for most biomedical applications where deeper tissue imaging in relatively high resolution is required. For example, diffuse optical tomography (DOT) has been extensively studied for detection of deep tissue abnormalities such as breast cancer and neonatal brain hemorrhage, it spatial resolution is limited between $3 \mathrm{~mm}$ to $5 \mathrm{~mm}$ for typical centimeter imaging depth [7]. The relative low spatial resolution hampers the DOT approach from further clinical application and diminishes its potential in diagnostic medicine, although rich optical scattering/absorption contrast together with key physiological changes (hemoglobin, oxygenation, etc) has been observed during early cancer development and hemorrhage onset.

In contrary to strong optical scattering in biological tissues, ultrasound scattering is two to three orders weaker in biological tissues $[8,9]$, therefore ultrasound wave is capable of providing enhanced signal to noise ratio (SNR) and higher spatial resolution over diffusive photon wave for objects located deep in biological tissues. By detecting ultrasound signals generated from lighted biological tissues, the hybridized photoacoustic imaging provides ultrasonically resolved optical absorption feature of biological tissues instead of mechanical properties such as tissue density in ultrasonography. For example, different optical absorbing organs (livers, kidney, spinal cord, etc) in a nude mouse and the optical absorption map of a human finger joint, which are both centimeter deep, can be visualized in sub millimeter resolution with photoacoustic imaging $[10,11]$ and higher resolution up to 100 micron has been achieved for photoacoustic imaging of high optical absorbing blood networks in a rat brain $[12,13]$. As one of the key optical biomarkers in tumor detection, optical absorption provided by photoacoustic imaging is an independent imaging contrast absent from other imaging modalities such as ultrasonography, $\mathrm{x}$-ray based radiography, OCT and Magnetic resonance imaging (MRI). The optical absorption biomarker is highly sensitive to tumor/rheumatoid development and can be as

*Corresponding author: Yao Sun, Department of Radiology, Translationa Imaging, Methodist Hospital Research Institute, Houston, TX 77030, E-mail: ysun@tmhs.org

Received July 31, 2011; Accepted September 12, 2011; Published September 22, 2011

Citation: Sun Y, Jiang H, O’Neill BE (2011) Photoacoustic Imaging: An Emerging Optical Modality in Diagnostic and Theranostic Medicine. J Biosens Bioelectron 2:108. doi:10.4172/2155-6210.1000108

Copyright: (c) 2011 Sun Y, et al. This is an open-access article distributed under the terms of the Creative Commons Attribution License, which permits unrestricted use, distribution, and reproduction in any medium, provided the original author and source are credited. 
high as 3:1 between normal tissues and tumor tissues in near infrared region. Furthermore, tissue optical absorption comes from different molecular components (oxy-hemoglobin, deoxy-hemoglobin, fat, lipids, water, etc), which have different absorption spectrum in typical optical diagnostic/therapeutic window (visible/near infrared light). By using multi-spectral light, physiological/functional parameters (hemoglobin concentration, oxygenation, water content, etc) can be further revealed to quantify tumor angiogenesis and hypoxia state, which are key biological markers for tumor development and critical for molecular tumor imaging and evaluation of targeted cancer treatment. In the past decade, thousands of papers have been reported to advance singleor multi-spectral photoacoustic imaging techniques and applications of photoacoustic imaging to diagnostic medicine, chemistry and genetics. Up to now, photoacoustic imaging has shown its potential to detect breast / oral / prostate / ovarian cancer, to assess vascular / skin / ocular / lymphatic diseases, to monitor epilepsy in small animals, to image finger joints and arthritis, to visualize fluorescent proteins, to evaluate exogenous contrast agents in molecular cancer imaging, to detect circulating tumour cells, to image biomarker in small animal model, etc. [10-13], [14-41]. In this paper, we provide a review of the photoacoustic imaging technique for studies in diagnostic and theranostic medicine. The technical consideration for photoacoustic imaging systems and reconstruction algorithms of structural / quantitative photoacoustic imaging will be introduced in section of "The Principle and System of Photoacoustic Imaging" and section of "Reconstruction Algorithms of Photoacoustic Imaging". Photoacoustic detection of breast cancer and arthritis will be discussed in section of "Photoacoustic Applications in Diagnostic Medicine" for possible photoacoustic applications in diagnostic medicine, followed by a brief review of optical controlled drug release and photoacoustic evaluation in section of "Photoacoustic Application in Theranostic Medicine" in this paper.

\section{The rinciple and System of Photoacoustic Imaging}

The fundamental physics beneath photoacoustic imaging is photoacoustic effect [1-3], where optical energy is converted into acoustic energy as a result of optical absorption and thermal expansion. As shown in the schematic of photoacoustic effect used for photoacoustic imaging in biomedicine (Figure 1), biological tissues are lighted by nanosecond laser pulses, which leads to the localized rapid temperature rise (several millidegrees) and the generation of wideband ultrasound pulses due to thermal expansion. The light excited ultrasound pulses propagate in the biological tissues and can be detected on a boundary surrounding the biological tissues by typical ultrasonic transducers used in ultrasonography.

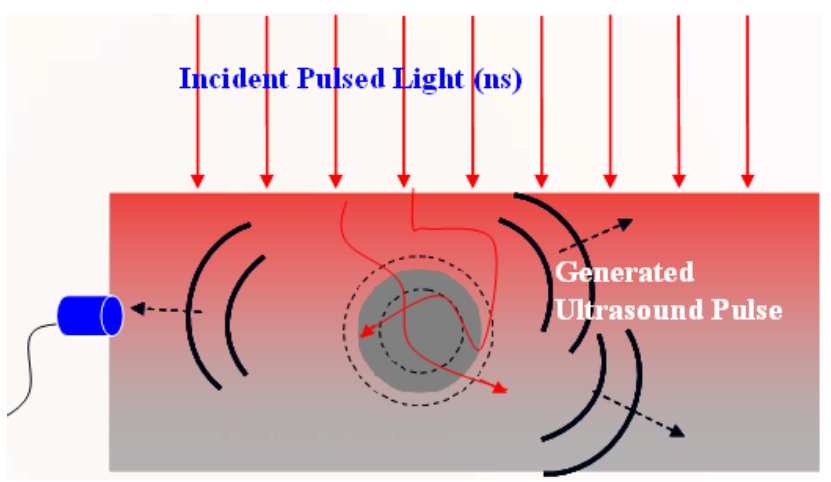

Figure 1: Schematic of photoacoustic effect.
For pulse mode photoacoustic generation propagating in soft tissues at room temperature, the thermal diffusion during the incident nanoseconds pulses is only around ten microns and is ignorable for most biomedical applications [42]. Therefore, only considering the thermal expansion mechanism, the photoacoustically generated acoustic field in tissue is described by the following wave equation:

$$
\left[\nabla^{2}-\frac{1}{c^{2}} \frac{\partial^{2}}{\partial t^{2}}\right] p(\vec{r}, t)=-\frac{\beta}{C_{P}} \frac{\partial}{\partial t}[\Phi(\vec{r}) I(t)]
$$

Where $c$ is the speed of acoustic wave in the tissue; $\beta$ is the thermal expansion coefficient; $\mathrm{C}_{\mathrm{p}}$ is the specific heat at constant pressure; $I(t)$ is the temporal profile of laser pulse. $p(\vec{r}, t)$ is the generated acoustic pressure wave and $\Phi(\vec{r})$ is the optical energy absorbed in the tissue, which is the product of tissue absorption coefficient $\mu_{a}(\vec{r})$ and optical fluence $\phi(\vec{r})$.

The major task in photoacoustic imaging is to recover the structural maps of deposited optical energy $\Phi(\vec{r})$ or quantitative distribution of absorption coefficient $\mu_{a}(\vec{r})$ in biological tissues from the governing equation (1), by acquiring the generated and propagated ultrasound wave $p(\vec{r}, t)$ with a photoacoustic imaging system. A prototype of photoacoustic imaging system to generate the photoacoustic signals in biological tissues and acquire the ultrasound signals for further image reconstruction can be illuminated with a developed system as shown in Figure 2. A typical photoacoustic imaging system is composed of lighting system, signal detecting system, object placement system and computer control system. A pulsed laser beam generated from a high power solid state laser (Yd: YAG, Ti: Sapphire, etc) is guided and expanded to illuminate the objects of interest. The duration of the laser pulse is around 10ns, which limit thermal diffusion and ultrasonic propagation to $10-15$ microns during the pulse. The repetition rate of the pulse is around $10-50 \mathrm{~Hz}$ for typical photoacoustic imaging systems. Although higher repetition rate is beneficial for real-time imaging or tissue dynamics study, thermal superheating of tissues may occur for pulse over $100 \mathrm{~Hz}$ [3]. The wavelength of the laser is variable from 532 to 1064 $\mathrm{nm}$ for maximized absorption of endogenous / exogenous targets and deeper tissue penetration in most reported studies, although UV light has also been used to image cell nuclei [43]. The laser pulse is guided by a lens-mirror system or optical fibers and is expanded to uniformly illuminate the tissues to be imaged in most applications, however the recently developed photoacoustic endoscope and optical-resolution microscope require very small laser spot or highly focused laser spot in stead of an area laser beam [43-49]. Due to laser safely, the light incident

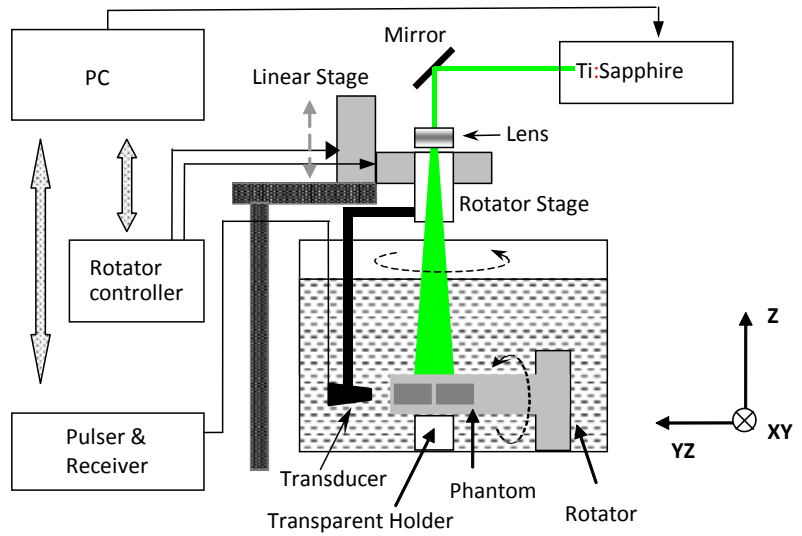

Figure 2: Schematic of photoacoustic imaging system. Reproduced with permission (Figure 1 of Reference 42). 
on the tissue surface should be controlled below $22 \mathrm{~mJ} / \mathrm{cm}^{2}$. Although high power solid state laser is commonly used in photoacoustic imaging, much smaller and cheaper diode laser can also be used as a lighting source in certain applications $[38,50,51]$, which may greatly reduce the cost of the photoacoustic imaging system and make it eligible for a carry-on diagnostic system in special situations such as point of care.

For photoacoustic imaging systems, the most critical part is the detecting system, which can be an ultrasound detecting array in an optimized geometry or a single broadband transducer controlled by step motors. In theory, the spatial resolution of the photoacoustic imaging system depends on both the detecting geometry and the transducer performance, where the axial (in-depth) and lateral spatial resolutions of a transducer are limited by its bandwidth and aperture size, respectively [52]. The ideal detecting system for photoacoustic imaging should be with broadband point detectors along a closed detecting surface surrounding tissues of interest, which allows the ultrasound signals from each acoustic source --optical absorber-- detectable at every boundary point without signal distortion. However, all ultrasound detectors in reality have finite-size active element and limited bandwidth, and only part of a closed detecting surface might be available in most real applications due to the limited access to certain surface of the objects (phantom, tissue, animal, organ, etc) to be imaged. The loss of a closed detecting surface results in incomplete data set for reconstruction procedure (limited view) and the reality of finite-size and limited bandwidth transducer result in the inevitable loss of lateral spatial resolution and possible loss of axial spatial resolution, if the bandwidth of the generated photoacoustic signals is not fully covered by the bandwidth of the detecting transducer. Besides, very small aperture or miniaturized transducer always result in greatly reduced signal sensitivity and deteriorated SNR level as well as elevated cost in the photoacoustic imaging system. As such, flat transducers with finite aperture size combined with optimized detecting geometry were commonly used in practice as an optimized photoacoustic imaging solution for moderate SNR level and cost at the expense of acceptably reduced lateral spatial resolution [11-14].

Analytical study [52] has shown that limited bandwidth of transducer restrains the spatial resolution (both axial and lateral) of photoacoustic imaging to around $0.6-0.8 \lambda_{c}$, where $\lambda_{c}$ is the wavelength of transducer high cutoff frequency $f_{c}$. The loss of low frequency components in detected photoacoustic signals does not blur the objects but may result in oscillating artifacts surrounding the reconstructed objects. It is worth to note that the above spatial resolution limitation is under the assumption that the high cutoff frequency of generated photoacoustic signals is higher than the transducer high cutoff frequency, otherwise $f_{c}$ should be the high cutoff frequency of photoacoustic signals to calculate the real spatial resolution limitation. For flat transducer with a diameter $A$, the limitation of lateral spatial resolution in a spherical detecting geometry gradually change from 0 to A when the object moves from the spherical center to the detecting location, while the lateral spatial resolution in a planar detecting geometry is always limited by A everywhere in the imaging region. The lateral spatial resolution in a cylindrical detecting geometry is the combination between that in spherical and planar detecting geometry, which means that it gradually change from 0 to $A$ when the object moves from the cylindrical axis to the cylindrical surface and it remains $A$ along the cylindrical axis. Compared to the lateral spatial resolution, the finite size of transducer can have a negligible effect on the axial resolution if the transducer size is much smaller than the detecting distance off the imaging center, since it is proportional to $0.25 \mathrm{~A}^{2}$ but inversely proportional to the distance of the detector from the imaging center. Previous experimental study [42] has also confirmed that a flat transducer in the spherical detecting provides volumetrically enhanced spatial resolution over cylindrical detecting geometry.

Cylindrical or spherical focused ultrasound transducer, the lateral resolution of which depends on the focal diameter of the transducer, has been adopted in certain photoacoustic applications to improve the lateral spatial resolution around the focal zone in the imaging plane/ volume $[12,34]$, particularly for near field photoacoustic imaging where targets of interest are very close to the detecting surface resulting in limited signal acceptance on the detecting surface and extremely worsened lateral spatial resolution with a flat transducer. In photoacoustic microscopy, spherical focal transducer seems the only option to achieve extremely high lateral spatial resolution ( $\sim 100$ microns), although the lateral spatial resolution drops quickly outside the focal zone and the raster scanning procedure is generally slow (one raster scan line corresponds only one pixel in the image). As same as in ultrasonography, a phase controlled ultrasound array [53,54] provide multiple focal zones or variable focal zone to improve lateral spatial resolution for target located in the far field of the ultrasound detectors.

In most biomedical applications, only part of a closed detecting surface is accessible and only incomplete data set is available leading to limited view reconstruction. Simulation study [55] has shown that objects inside of the "invisible" domain, which is defined as a domain where any line connecting two virtual points along the missing experimental detecting loci/surfaces does not cross the accessible detecting loci/surfaces, may be blurred during the reconstruction while objects inside of the "detection region" a complement to the "invisible" domain can be "stably" reconstructed. Specifically, for the objects inside of the "invisible" domain, "the parts of the boundaries the normal lines to which pass through a detector position" can be stably reconstructed while other boundaries will blur away during the reconstruction. Because of this limitation from a partial detecting surface (limited view), most photoacoustic imaging systems use open arc or open spherical detecting geometry to maximally cover the objects of interest in a "detection region" [10-11, 20-21,56-58]. In some situations for example, reflection mode photoacoustic imaging of skin diseases where the objects of interest is outside of the "detection region" of the accessible detecting surfaces, a focused transducer or transducer array with focusing capability around the objects might be an alternate solution to improve the spatial resolution in photoacoustic imaging.

Although PZT transducers have been commonly used in photoacoustic imaging, PVDF and Fabry Perot (FP) polymer film ultrasound sensor have also been studied to improve the detector performance under certain biomedical applications [13,19,20,43,59-63]. Although the sensitivity of PVDF is about two-order lower than PZT material, PVDF transducer has wider bandwidth for high resolution imaging and shape flexibility for curved detecting array. FP polymer film is optically transparent [61] and the light to excite the photoacoustic signals in the tissues is able to pass through the FP sensor to the objects of interest, without inducing any background ultrasound signals from the detecting system. This unique character makes FP sensor a most appealing ultrasound detecting system for reflection mode photoacoustic imaging or contact detecting for targets near the detecting surface. Three dimensional photoacoustic imaging of the vasculature in a mouse brain has been reported with reflection-mode and contact detection based on FP polymer film. As shown in Figure 3(a), the FP sensor head is placed on the surface of the mouse head and the nanosecond excitation laser pulses are directed on to the sensor head and transmitted through it 
into the underlying tissue (mouse head). The cerebral vascular anatomy can be visualized with high contrast and spatial resolution to depths up to $3.7 \mathrm{~mm}$, as shown in Figure 3(b). It is claimed that this detecting system may be applicable in characterizing small animal models of human disease and injury processes such as stroke, epilepsy, and traumatic brain injury.

\section{Reconstruction Algorithms of Photoacoustic Imaging}

Thus far, several algorithms have been implemented to effectively reconstruct photoacoustic images from measured acoustic waves, such as back-projection, Fourier transform, P-transform, k-wave method, statistical approaches and finite element based reconstruction [64-71]. While others rely on analytical solutions to the photoacoustic wave equation in a regularly shaped imaging domain and assume acoustical homogeneity in biological media, finite element based algorithm is capable to accommodate tissue heterogeneity and geometric irregularity as well as allow complex boundary conditions and source representations. Acoustic property in the biological tissues is also able to be recovered simultaneously with optical property by finite element based algorithm, when acoustic heterogeneity is taken in consideration in photoacoustic wave equation. Moreover, finite element based algorithms share the same frame for different problems, which allows photoacoustic reconstruction algorithm easily adapted / integrated with other imaging modalities for multimodal imaging or optical transport model for quantitative imaging to obtain physiological / functional information of the imaged tissues. As such, we focus on finite element based algorithms in this section for conventional photoacoustic imaging and single- / multi-spectral quantitative photoacoustic imaging.

\section{Reconstruction of optical energy deposit and simultaneous reconstruction of acoustic heterogeneity}

Finite element based reconstruction algorithm for conventional photoacoustic imaging uses a regularized Newton iterative strategy to update an initially estimated optical/acoustic property distribution to minimize an object function, which is composed of a weighted sum of the squared difference between computed and measured acoustic data. The computed acoustic data is obtained by solving the following photoacoustic wave equation [69], which is a Fourier transformed equation in the frequency domain from the equation (1) in the temporal domain, subject to the second-order absorption boundary conditions using finite element method (FEM):

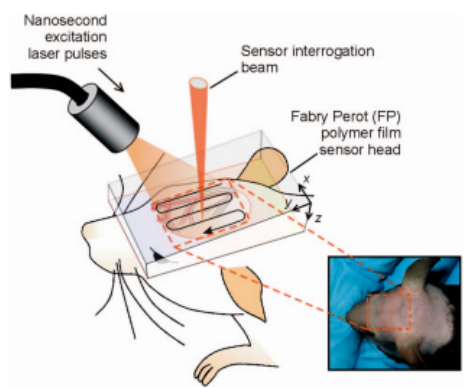

(a)

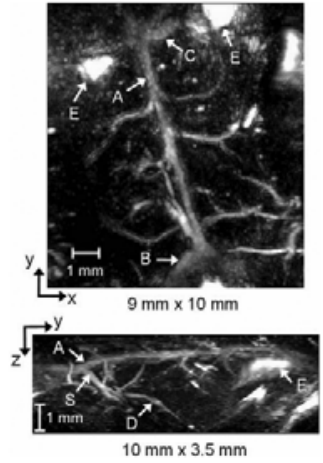

(b)
Figure 3: Experimental arrangement (a) and reconstructed images (b) in photoacoustic imaging of the vasculature in the mouse brain based on FP sensor. $A$, superior sagittal sinus $B$, transverse sinus; $C$-inferior cerebral vein; $E$, eyes; $S$, sinus rectus; $D$, inferior sagittal sinus. Reproduced with permission (Figure 1 and 4 of Reference 13).

$$
\nabla^{2} P(\vec{r}, \omega)+k_{0}{ }^{2}(1+O(\vec{r})) P(\vec{r}, \omega)=i k_{0} \frac{c_{0} \beta \Phi(\vec{r})}{C_{p}}
$$

where $\mathrm{P}$ is the pressure wave; $k_{0}=\omega / c_{0}$ is the wave number described by the angular frequency, $\omega$ and a reference (average) speed of the acoustic wave in the medium, $c_{0}: O$ is a coefficient that depends on both acoustic speed and attenuation as follows.

$$
O(\vec{r})=\frac{c_{0}^{2}}{c^{2}(\vec{r})}-1+\frac{i}{k_{0}} \frac{A c_{0}}{c^{2}(\vec{r})}
$$

where $\mathrm{c}$ is the speed of the acoustic wave in the medium on tissue; $\mathrm{A}$ is the acoustic attenuation coefficient;

The core equations for forward and inverse computations in finite element based reconstruction can be written as the following linear equations, by taking finite-element discretization on equation (2)

$$
\begin{aligned}
& {[A]\{p\}=[B]\{\phi\}} \\
& \left(\mathfrak{J}^{\mathbf{T}} \mathfrak{I}+\lambda \mathbf{I}\right) \Delta \chi=\mathfrak{J}^{T}\left(p^{m}-p^{c}\right)
\end{aligned}
$$

where the elements of $[\mathrm{A}]$ and $[\mathrm{B}]$ are and $A_{i j}=\left\langle\nabla \psi_{j} \cdot \nabla \psi_{i}-k_{0}^{2}\left[1+\sum_{l} O_{l} \psi_{l}\right] \psi_{j} \psi_{i}\right\rangle_{V}-\left\langle\psi_{i}\left(\nabla \psi_{j} \cdot \hat{n}\right)\right\rangle_{S} . B_{i j}=-i k_{0} c_{0} \beta\left\langle\psi_{i} \psi_{j}\right\rangle_{V} / C_{P}$ \langle\rangle$_{V}$ and \langle\rangle$_{S}$ indicate the volume and surface integration over the problem domain and boundary, respectively. $\psi_{i}$ and $\psi_{j}$ are Lagrangian basis function at node $i$ and $j$, respectively. $p=\left\{p_{1}, \cdots, p_{N}\right\}^{T}$ and $\phi=\left\{\phi_{1}, \cdots, \phi_{N}\right\}^{T}$ are the acoustic pressure and absorbed optical energy density at each node. $\Delta \chi=\left\{\Delta O_{1}, \cdots, \Delta O_{N}, \Delta \phi_{1}, \cdots, \Delta \phi_{N}\right\}^{T}$ is the update vector for the acoustic heterogeneity and absorbed optical energy density. $p^{m}=\left\{p_{1}^{m}, \cdots, p_{M}^{m}\right\}^{T}$ and $p^{c}=\left\{p_{1}^{c}, \cdots, p_{M}^{c}\right\}^{T}$ are the measured and calculated acoustic pressure for $\mathrm{i}=1,2 \ldots, M$ boundary locations. $\mathfrak{I}$ is the Jacobian matrix formed by $\partial p / \partial \chi$ at the boundary measurement sites. $\lambda$ is the regularization parameter determined by combined Marquardt and Tikhonov regularization schemes.

With the above reconstruction algorithm, a simulation case can be tested. As shown in Figure 4(a), the target A has an optical contrast (optical deposited energy) of 2:1 without acoustic contrast; the target B has a acoustic contrast (speed of sound) of 1560:1485 without optical
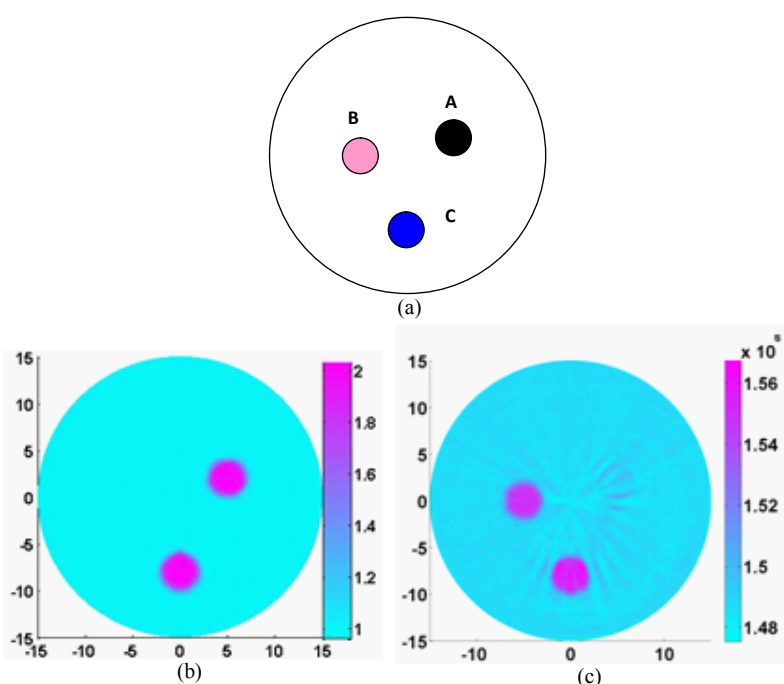

Figure 4: Simulation geometry (a) and simultaneously reconstructed absorbed optical energy (b) / acoustic heterogeneity (c) by finite element based photoacoustic reconstruction algorithm considering acoustic heterogeneity. The optical contrast for target A, B, C is $2: 1,1: 1$ and $2: 1$, respectively. The acoustic contrast (speed of sound) for target A, B, C is 1485:1485, 1560:1485 and 1560:1485, respectively. 
contrast; the target $\mathrm{C}$ has both acoustic contrast of 1560:1485 and optical contrast of 2:1. 5\% white noise was introduced into the simulated data. After several iterations (5 times), targets with different contrast can be separated from each other and results in well recovered optical property as well as acoustic heterogeneity in the targets, as can be observed in Figure 4(a-b).

In most photoacoustic applications, acoustical homogeneity has been assumed for simplicity in the reconstruction procedure. In this situation, the acoustical-heterogeneity based reconstruction algorithm (2-5) can degrade to acoustical-homogeneity based reconstruction algorithm to reconstruct optical contrast only, by removing the acoustic heterogeneity part $O$ from equation (2-5).

\section{Recovery of optical absorption coefficient with single wave- length light}

The conventional photoacoustic images depict the maps of deposited optical energy $\Phi(\vec{r})$ in the tissue, which is the product of intrinsic tissue absorption coefficient $\mu_{a}(\vec{r})$ and optical fluence $\phi(\vec{r})$. By combining conventional photoacoustic imaging technique with photon transportation model, it is feasible to further recover the intrinsic optical absorption coefficient $\mu_{a}(\vec{r})$. The quantitative recovery is obtained by using the following photon diffusion equation as well as the Robin boundary,

$$
\begin{aligned}
& \nabla \cdot D(r) \nabla(E(r) \Phi(r))-\Phi(r)=-S(r) \\
& -D \nabla(E(r) \Phi) \cdot n=E(r) \alpha \Phi
\end{aligned}
$$

where $E(r)=1 / \mu_{a}(r), D(r)$ is the diffusion coefficient, $D=1 /\left(3\left(\mu_{a}+\mu_{s}^{\prime}\right)\right)$ and $\mu_{s}^{\prime}$ is the reduced scattering coefficients, $\alpha$ is a boundary condition coefficient related to the internal reflection at the boundary, and $S(r)$ is the incident point or distributed source term.

One approach to recover the optical absorption coefficient is through a forward fitting procedure, starting from an estimated distribution $\mu_{a}(\vec{r})$ which is an optimized initial based on the results of a searching scheme for optimal initial. The reduced scattering coefficient $\mu_{s}^{\prime}$ is approximated as a pre-known constant for simplicity. The optical fluence $\phi(\vec{r})$ and the absorbed energy density $\Phi^{c}$ are iteratively calculated through the forward model of photon diffusion equations (6-7) and the relation $\Phi^{c}(\vec{r})=\mu_{a}(\vec{r}) \phi(\vec{r})$. If the error between $\Phi$ and $\Phi^{c}$ is not small, then $\mu_{a}(\vec{r})$ is updated by $\mu_{a}(\vec{r})=\Phi(\vec{r}) / \phi(\vec{r})$ and the above procedure is repeated until a small error between $\Phi$ and $\Phi^{c}$ is reached, resulting in a stable quantitative distribution of $\mu_{a}(\vec{r})$.

Another approach is to inversely recover the optical absorption coefficient by introducing a prior structural information to regularize the inverse solution and iteratively finding the global optimized distribution of optical absorption coefficient $\mu_{a}(\vec{r})$. In this approach, $E(r)$ which is the reciprocal of $\mu_{a}(\vec{r})$ is updated by the following equation

$$
\Delta(\mathbf{E})=\left(\mathbf{J}^{T} \mathbf{J}+\lambda \mathbf{I}+\mathbf{L}^{T} \mathbf{L}\right)^{-1}\left[\mathbf{J}^{T}\left(\boldsymbol{\Phi}^{o}-\boldsymbol{\Phi}^{c}\right)\right]
$$

Here, the PAT image (absorbed optical energy density) is used both as input data and as a prior structural information which is incorporated into Laplacian-type filter matrix L [79].

It is worth to note that the scattering coefficient is assumed as constant/homogeneous for the use of the above algorithms to quantitatively recover the optical absorption coefficient. This assumption suggests that the above quantitative reconstruction procedure may be applicable to cases where the scattering contrast is low or the objects having scattering contrast are small in size.

\section{Quantitative photoacoustic imaging with multi-spectral light}

In biological tissues, the optical absorption comes from different tissue chromophores (oxy-hemoglobin, deoxy-hemoglobin, fat, lipid, water, etc), which have different optical absorption spectrum. The overall optical absorption is determined by the following Beer's law in consideration of the optical absorption contributions from L chromophores.

$$
\mu_{a}(\lambda, \vec{r})=\sum_{i=1}^{L} \delta_{i}(\vec{r}) \varepsilon_{i}(\lambda)
$$

Where $\delta_{i}(\vec{r})$ is the concentration of $i^{\text {th }}$ chromophore in the unit of molar $M($ mole $/ L) ; \varepsilon_{i}(\lambda)$ is the absorption extinction coefficient of the $i^{\text {th }}$ chromophore at wavelength $\lambda$. By replacing $\Phi(\vec{r})$ at each optical wavelength in equation (2) with $\Phi(\vec{r})=\mu_{a}(\vec{r}) \phi(\vec{r})$ and the relation in Beer's law, we have the following multi-spectral based photoacoustic equation [78]

$$
\cdot \nabla^{2} P(\vec{r}, \varpi, \lambda)+k_{0}^{2}(1+O(\vec{r})) P(\vec{r}, \varpi, \lambda)=i k_{0} \frac{c_{0} \sum_{1}^{L} \delta_{i}(\vec{r}) \varepsilon_{i}(\lambda) \phi(\vec{r}, \lambda)}{C_{P}}
$$

By combining the above multi-spectral photoacoustic equation (1) with the photon diffusion equation (6-7), which is used to update the optical fluence $\phi(\vec{r}, \lambda)$ from the iteratively updated chromophore
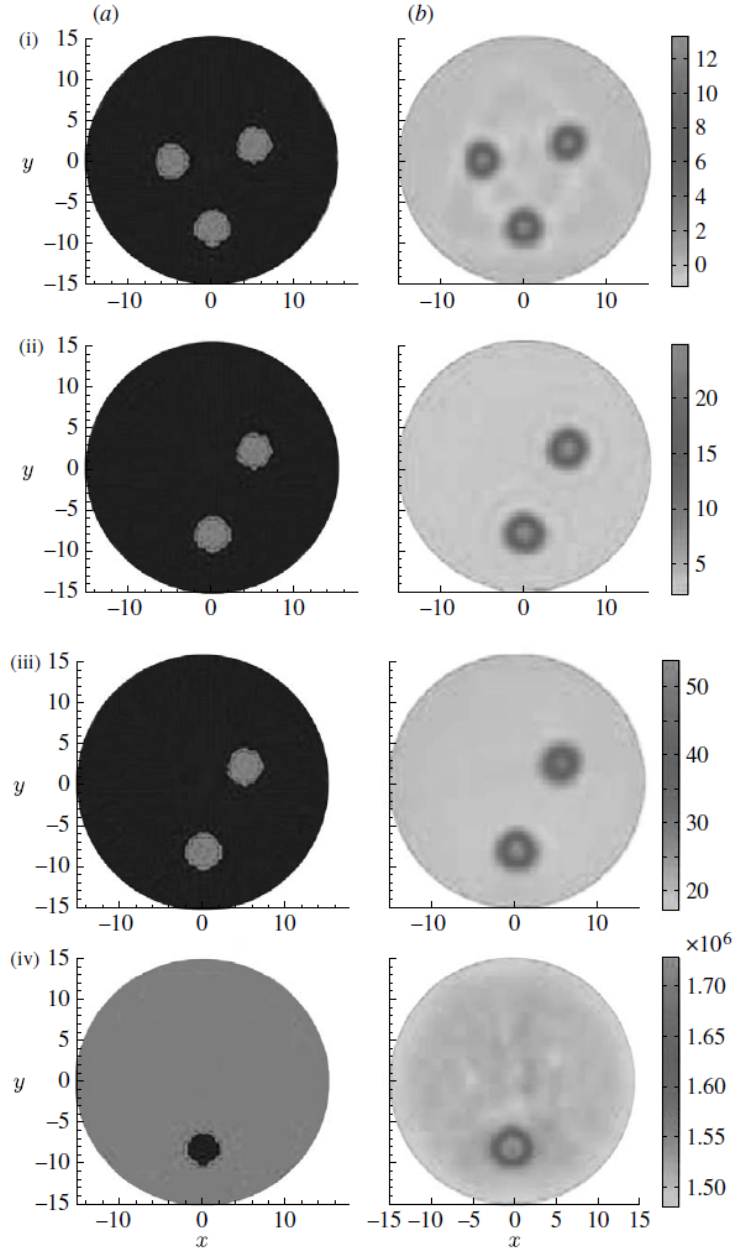

Figure 5: Exact (a) and reconstructed images (b) using multi-spectral photoacoustic reconstruction algorithm. The first to the fourth rows show (i) $\mathrm{Hb}(\mathrm{mM})$ (ii) $\mathrm{HbO} 2$ (mM), (iii) $\mathrm{H}_{2} \mathrm{O}(\%)$ and (iv) acoustic velocity ( $\left.\mathrm{mm} / \mathrm{s}\right)$ images, respectively. Reproduced with permission (Figure 2 of Reference 11). 
concentrations (therefore the combined absorption coefficient), the acoustic heterogeneity and each chromophore concentration can be iteratively reconstructed by following the similar reconstruction procedure described in section "Reconstruction of optical energy deposit and simultaneous reconstruction of acoustic heterogeneity".

Simulation studies based on the above quantitative photoacoustic imaging with multi-spectral light have been reported [78]. Six optical wavelengths $(633,682,723,850,854$ and $930 \mathrm{~nm})$ and a circular background ( $15 \mathrm{~mm}$ in radius) containing three circular targets $(2 \mathrm{~mm}$ in radius each), as shown in Figure 5(a), has been tested with the developed algorithm. $2 \%$ random Gaussian noise was introduced to the simulation data. As shown in Figure 5(b), four parameters $\left(\mathrm{Hb}, \mathrm{HbO}_{2}\right.$, $\mathrm{H}_{2} \mathrm{O}$ and acoustic velocity) have been spatially resolved by multi-spectral reconstruction algorithm of photoacoustic imaging.

Studies has shown that the quantitative evaluation of these tissue chromophores and their physiological / functional changes are of great significance both in basic science (such as neuroscience, molecular imaging of small animals) and clinical applications (such as breast cancer detection and classification). Photoacoustic imaging is a high resolution imaging modality for optical contrast; as such the multispectral quantitative photoacoustic imaging might be potential for high resolution tissue chromophores imaging/measurement

\section{Photoacoustic Applications in Diagnostic Medicine}

Up to now, photoacoustic imaging has been extensively studied as clinical / preclinical tool to detect cancer and evaluate disease in different organs. In this section, we focus on a review of the photoacoustic imaging in breast cancer detection and osteoarthritis evaluation, where patient subjects have been already recruited and photoacoustically evaluated. Preclinical photoacoustic studies will also be touched at the end of this section.

\section{Photoacoustic detection of breast cancer}

Breast cancer is the most commonly diagnosed non-skin cancer and the leading cause of cancer death for women. The American Cancer Society suggests a yearly clinical breast examination by a health care provider and mammography every year from age 40 years. However the X-ray mammography, which is the only clinically available tool for mass screen of breast cancer, requires an ionizing radiation $\mathrm{X}$-ray source and poses harm to patients. Furthermore, X-ray mammography has limited sensitivity and specificity, which results in small tumor undetectable at the early stage and 4-5 benign cases need undergo breast biopsy for every cancer detected by X-ray mammography [81-84]. At the same time, high absorption contrast between tumor and normal tissues has been observed, which is up to 3:1 in near infrared region; and oxy-hemoglobin level differences between tumor and normal tissues can be up to $4: 1$. The high optical contrast existing in the breast cancer and higher spatial resolution capability of photoacoustic imaging for up to 5-7 centimeter penetration depth enable photoacoustic imaging a promising safe tool for routine breast cancer screen.

Since last decade, extensive studies have been conducted in photoacoustic imaging of breast cancer, advancing both the imaging systems and the clinical studies. In pilot studies, a laser optoacosutic imaging system (LOIS) comprised of an arc-shaped 32 PVDF ultrasound detecting elements was developed to detect breast cancer in vivo. Each element is $1 \mathrm{~mm} \times 12.5 \mathrm{~mm}$ with a $3.85 \mathrm{~mm}$ gap from the adjacent element. The arc where the 32 elements are evenly arranged covers $120^{\circ}$ with a radius of $60 \mathrm{~mm}$, which allows $60 \mathrm{~mm} \times 60 \mathrm{~mm}$ field of view. The lateral spatial resolution is $1-2 \mathrm{~mm}$ in the entire field of view. Pulsed light at two wavelengths ( $757 \mathrm{~nm}$ and $1064 \mathrm{~nm}$ ) was delivered by optical fibers to the examined sites. The light pulse has a $10 \mathrm{~ns}$ duration and $20 \mathrm{~Hz}$ repetition rate. The signal acquisition and image reconstruction based on backprojection take about 2 seconds in total for a two-dimensional image. With 32-element LOIS, a breast ductal-lobular cancer tumor, which is about $6 \mathrm{~mm} \times 7 \mathrm{~mm}$ and $11 \mathrm{~mm}$ beneath the breast surface, was detected in vivo. The LOIS is further improved in a recent study [20], where 27 patients has been clinically examined with the new system called LOIS-64. The new system has an arc-shaped imaging array (acoustic detector probe) comprised of 64 PVDF rectangular element $(3 \mathrm{~mm} \times$ $20 \mathrm{~mm}$ ) with $0.11 \mathrm{~mm}$ thick. The acoustic detector probe is shaped as a hemi-cylindrical cup with the radius of its cylindrical surface of 70 $\mathrm{mm}$ and width of $90 \mathrm{~mm}$ to image a single breast slice in craniocaudal or mediolateral projection. $757 \mathrm{~nm}$ pulsed light was delivered by optical fiber bundle and expanded to illuminate the surface of the breast. The laser beam on the breast was $70 \mathrm{~mm}$ in diameter had a maximum fluence of $10 \mathrm{~mJ} / \mathrm{cm}^{2}$. The light pulse has a $75 \mathrm{~ns}$ duration and $10 \mathrm{~Hz}$ repetition rate. The single-channel sensitivity of the system is $1.66 \mathrm{mV} / \mathrm{Pa}$ and the spatial resolution is at least $0.5 \mathrm{~mm} .27$ patients have been examined by LOIS-64 and 18 out of 20 malignant lesions suspected from mammography have been successfully detected.

Besides LOIS for breast cancer detecting, photoacoustic mammoscope (PAM) has also been developed in recent years for examine breast cancer in vivo. Pulsed light $(5 \mathrm{~ns}$ duration and $10 \mathrm{~Hz}$ repetition rate) from an Nd: YAG laser is used to illuminate the regions of interest (ROI) of the breast. A parallel plate geometry has been adopted in PAM, where the breast will be gently compressed between a glass plate. A flat ultrasound detector matrix comprised of 590 elements is adopted to record photoacoustic signals from regions of interest, where an appropriate number of the elements are activated in succession. Each of the 590 ultrasound detecting elements is $2 \mathrm{~mm} \times 2 \mathrm{~mm}$ square and arranged in a roughly circular grid with a centre-centre spacing of 3.175 $\mathrm{mm}$. The PAM provides 3D reconstructions using delay-and-sum beam forming algorithms, capable of imaging inhomogeneities of $2 \mathrm{~mm} \mathrm{di-}$ ameter down to $32 \mathrm{~mm}$ depths with resolutions of $3.5 \mathrm{~mm}$. The examination time is about $45 \mathrm{~min}$. which permits an area of $52 \times 52 \mathrm{~mm}$ to be scanned. 13 patients have participated in the PAM studies, 6 of which result in successful measurement. 5 of the 6 patients with successful measurement were on suspect breasts and 1 was on a breast with a cyst. PAM images of the 5 suspect breasts have revealed regions of higher absorption in ROIs attributable to tumor vascularization.

The major advantages of photoacoustic imaging of breast cancer are the high spatial resolution (up to $0.5 \mathrm{~mm}$ ), the sufficient imaging depth $(5-7 \mathrm{~cm})$, the rich optical contrast related with tumor angiogenesis and the spectroscopic capability to detect physiological / functional (hemoglobin level, oxygenation, water content, etc) changes associated with tumor development [77]. However the challenges remain in the photoacoustic imaging of breast cancer, due to large volume of breast tissue and efficient light penetration to several centimeter deep with acceptable SNR level in the generated photoacoustic signal. Transducer array with more amount of ultrasound detecting elements for enlarged field of view and higher sensitivity for efficiently detecting weak photoacoustic signals from deeper breast tumor may help advance the photoacoustic imaging to further clinical application.

\section{Photoacoustic evaluation of osteoarthritis}

Osteoarthritis (OA) is the most prevalent degenerative joint disease, which involves tens of millions of Americans with disability or diminished quality of life. The typical pathological features of which include erosion of articular cartilage, bony changes and occasional synovial 
effusion as well as associated enhanced blood vessel growth [86-89]. Increased optical absorption coefficient (as well as optical scattering coefficient) in the diseased synovial membrane/fluid has been reported in both osteoarthritis and rheumatoid arthritis, due to increased turbidity and vascularization during the disease progress [90]. In fact, this optical increase can be as large as $100 \%$ at certain wavelengths in the NIR region [91-96]. As a hybridized imaging technique, photoacoustic may provide highly resolved images of the rich optical contrast associated with arthritis (osteoarthritis and rheumatoid arthritis) for economical and efficient detection of arthritic diseases.

Photoacoustic imaging of joint structure in animal models, cadaver finger and in vivo human subject have been reported [11,14-17] Different joint tissues in the proximal interphalangeal (PIP) and distal interphalangeal (DIP) joints from a cadaver human finger can be highly resolved by two-dimensional (2D) photoacoustic imaging in the cross section, including phalanx, volar plate, subcutaneous tissue, tendon and aponeurosis [14]. A later study adopted three dimensional spherical scan to improve the volumetric spatial resolution around the shape-heterogeneous joint cavity and optimized lighting geometry to avoid strong absorption from the blood vessels around the soft tissues (cartilage and synovial fluid) of interest in joint cavity [15]. An in vivo DIP joint from a human subject has been successfully imaged with the improved imaging system, where joint cavity/cartilage can be identified from the adjacent bones and the major arteries (lateral and median) as well as tendon can be identified from volumetric photoacoustic slices.

In a recent report, a quantitative three-dimensional photoacoustic approach has been evaluated with seven subjects (two OA patients and five healthy controls) enrolled in the study [11]. The ultrasound detecting system is an ultrasound detection array composed of eight $1 \mathrm{MHz}$ circular flat transducers combined with two rotary stages for a spherical scanning surrounding the finger joint to be imaged. Near-infrared

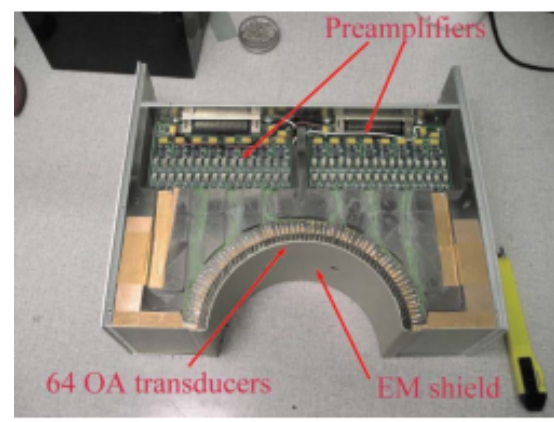

(a)

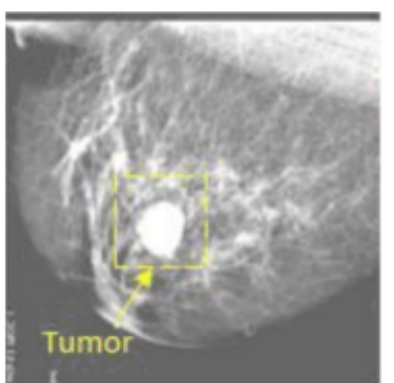

(b)

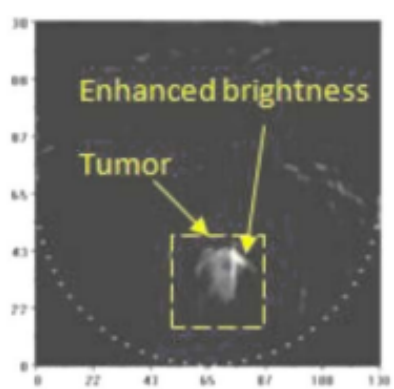

(c)
Figure 6: Optoacoustic probe for LOIS-64 (a) and mammography (b) / optoacoustic (c) image of the breast with a round benign tumor. Reproduced with permission (Figure 3 and 17 of Reference 20). light at $805 \mathrm{~nm}$ is guided and expanded to illuminate the dorsal side of the finger joint, which allow the maximal light penetration in finger tissues and best-resolved joint structures. The whole examination at one wavelength takes 5 minutes, covering 360 locations along the spherical surface. One DIP finger joint, joint II of the left hand, from each subject has been examined clinically and photoacoustically. Six of the seven recruited subjects resulted in successful photoacoustic image reconstruction while one healthy control failed due to the severe motion error of finger during the photoacoustic examination. As observed from the reconstructed results shown in Figure 7, the bones are clearly delineated from the adjacent tissues (cartilage and fluid) in the joint cavity, which is clearly identified on consecutive slices in both coronal and sagittal sections. The spatial resolution around the joint cavity is much better than the previously reported result, due to the reduced examination time leading to less motion error. The synovial fluid seems be differentiable from the surrounding cartilages as well in some reconstructed slices. From the measured thickness of the joint cavity (synovial fluid and cartilage), dimensional narrowing of the joint cavity is observed for OA joints (1.1-1.5mm for OA and 1.7-2.5mm for healthy joints). A quantitative analysis on all the photoacoustically examined patients further reveals that the ratio between volumetric mean absorption coefficient of the synovial fluid / cartilage and that of the bone is high in OA subjects and low in normal subjects $(0.58-0.63 / 0.72-0.76$ for OA joints verse $0.25-0.46 / 0.43-0.61$ for normal joints, respectively). The optical difference can be as large as 2:1 in the OA joints compared to the normal joints, if the ratio of the lowest sectional absorption coefficient of the joint cavity to the volumetric mean absorption coefficient of the bone is compared $(0.38-0.50$ for OA joints vs $0.11-0.26$ for normal joints).

Photoacoustic imaging of the joint cavity offers a sub-mm spatial resolution and has the potential to be further enhanced when larger amount of transducer elements and higher frequency transducers are used. As an emerging imaging technique, the advantage of photoacoustic imaging for finger joint imaging and detection of arthritis (osteoarthritis and rheumatoid arthritis) lies in its capability to provide highly resolved optical absorption coefficient which is an indicator for arthritic progress, acoustical properties in the joint structure when acoustic heterogeneity is considered in the reconstruction, and physiological/ functional parameters such as oxygenation and water content if multispectral light is used. Compared with other organs, finger joint is the most accessible for photoacoustic detecting and maximally avoids limited-view problem. In addition, photoacoustic imaging system is portable and low in cost. As such, photoacoustic imaging may provide a sensitive and affordable imaging method for early diagnostics of arthritis, monitoring of treatment response, and accelerating the advancement of medical therapies for arthritis.

In reported studies, homogenous acoustic approximation (constant acoustic speed) to the actual heterogeneous acoustic media of the finger joints has been assumed in the reconstruction, which may result in noticeable blur in the reconstructed image. Besides, the reflection and diffraction from the high density bone structure may distort the acoustic pressure wave and blur the reconstructed images as well. An advanced reconstruction model or appropriate correction of those effects may help improve the image quality of photoacoustic resolved finger joints and facilitate the further clinical evaluation of photoacoustic imaging in arthritis detection [72,96-99].

\section{Photoacoustic Application in Th ranostic Medicine}

Theranostics is an emerging interdiscipline field, which involves 

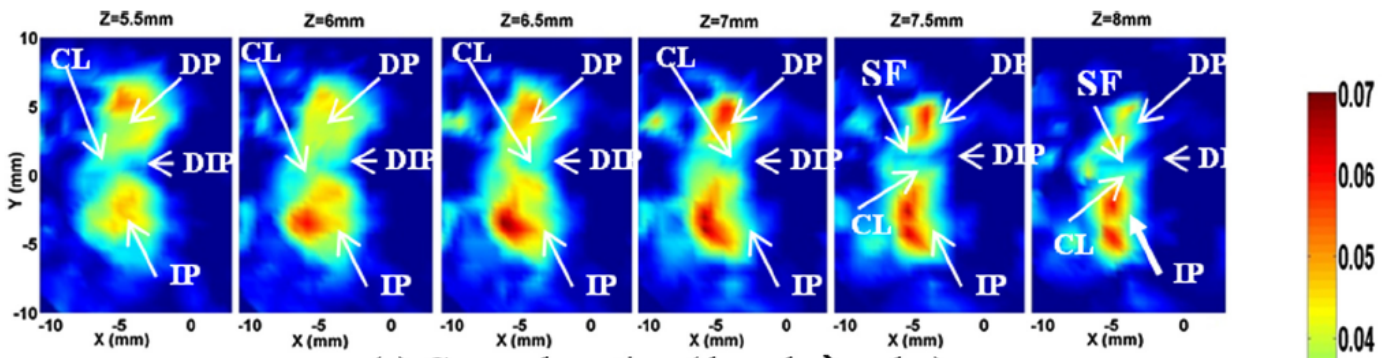

(a) Coronal section (dorsal $\rightarrow$ palm)

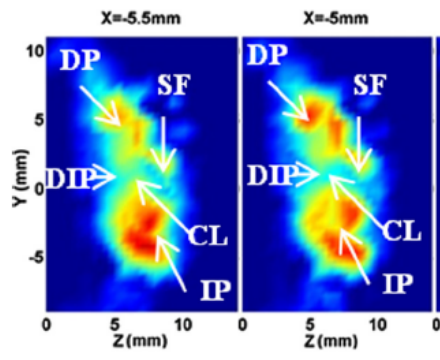

$X=-4.5 \mathrm{~mm}$
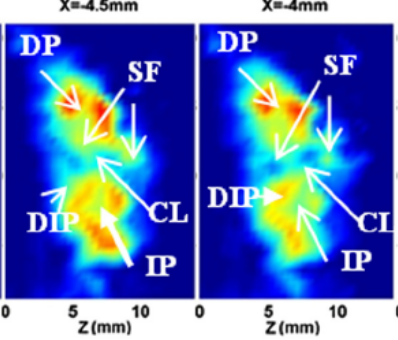

$X=-3.5 \mathrm{~mm}$

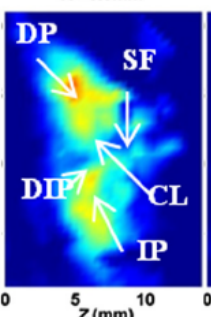

$X=-3 \mathrm{~mm}$

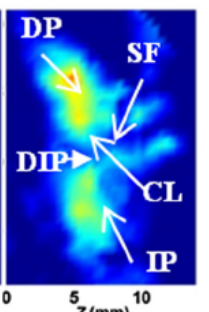

0.02
0.01
0

(b) Sagittal section (Medial $\rightarrow$ Lateral)

Figure 7: (a) Selected coronal and (b) sagittal planes of the recovered 3D absorption coefficient image for a typical normal joint. DP: distal phalanx; IP: intermediate phalanx; DIP: distal interphalangeal joint; CL: cartilage; SF: synovial fluid. Reproduced with permission (Figure 3 of Reference 79).

a)
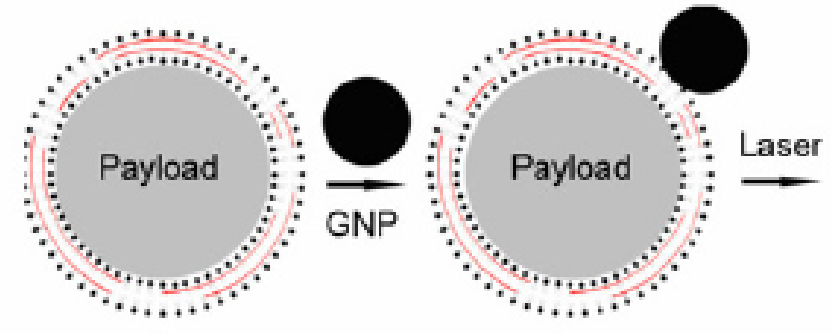
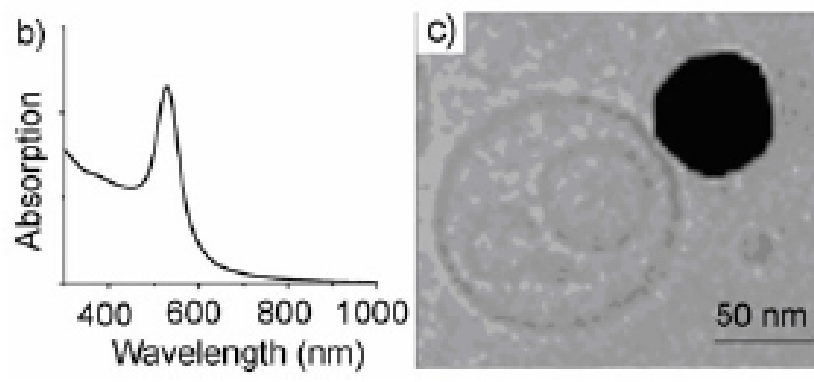

d)
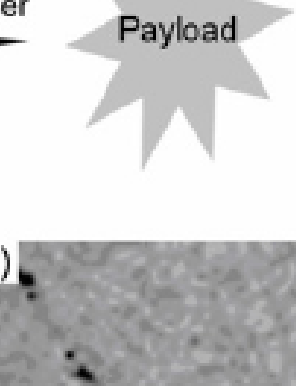

Figure 8: (a) Illustration of the releasing process; (b) UV-vis absorption spectrum of gold nanoparticles (GNPs); (c) a typical cryo-EM image of a PPL tethering a GNP; and (d) a typical cryo-EM image showing PPL/GNP complexes after laser irradiation. The scale bar in (c) also applies to (d). Reproduced with permission (Figure 3 of Reference 109).

disease diagnostics, treatment and evaluation of individual treatment response in a same platform. Thanks to the explosive advancement of nanotechnologies, different nano systems (liposome, polymeric micelle, nano droplet, metallic nano-sphere / rod / shell / cage, carbon nanotube, etc) have been developed to combine various functionalities in a same platform [100-107]. For example, chemotherapeutic drugs, imaging agents and targeting moieties can be combined together in the same nano system, which allows not only targeted treatment but also targeted imaging and evaluation of treatment response by appropriate imaging techniques. Well controlled drug delivery / release will allow maximized treatment efficiency of chemotherapy while greatly reduced side effect, as such internal stimuli ( $\mathrm{pH}$, hypoxia, enzymatic degradation, etc) and external energy (ultrasound and light) have been extensively studied for targeted drug delivery/release. Since photoacoustic imaging is a hybridized imaging technique combining both ultrasound and light, and photoacoustic imaging shares the same/similar lighting 


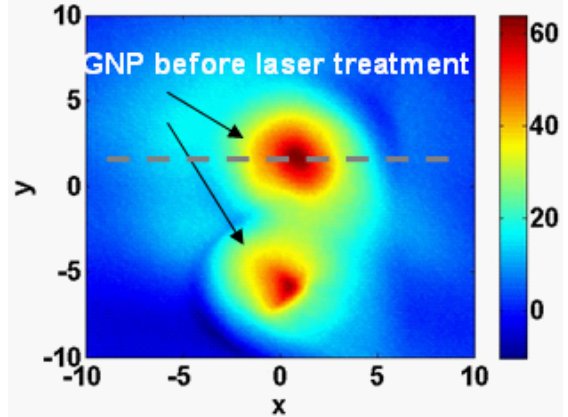

(a)

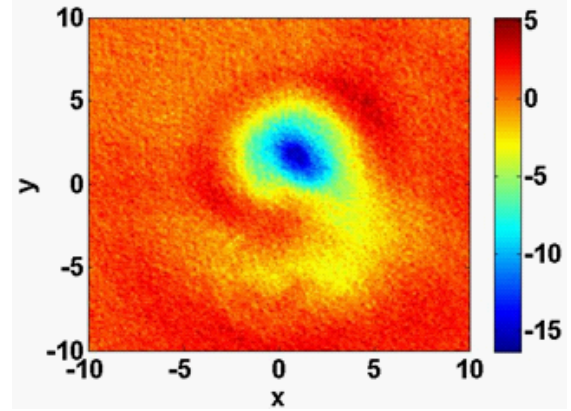

(c)

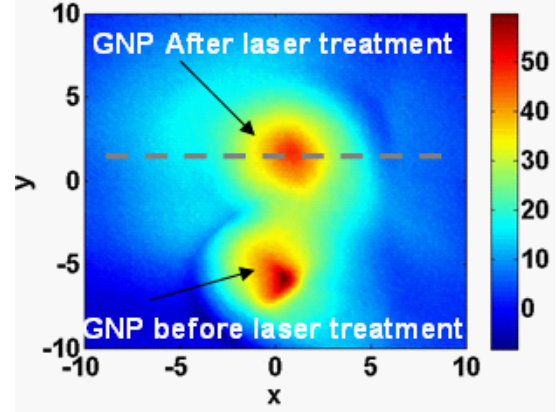

(b)

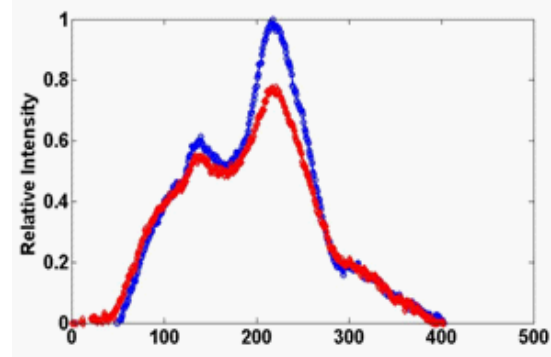

(d)

Figure 9: Photoacoustic evaluation of gold nanoparticles breakdown. (a) Photoacoustic image of GNP targets before high energy laser triggering (b) Photoacoustic image of GNP targets after high energy laser triggering (c) subtract image between photoacoustic images shown in (a) and (b) (d) Quantitative curves along the center of the evaluation target before/after high energy laser triggering of GNP solution.

system with light controlled drug release, it is interesting and promising to combine photoacoustic imaging with the targeted drug release in an integrated platform. Here, we provide a brief review of light controlled drug release system and a discussion of the possibility for integrated photoacoustic imaging with the targeted drug delivery / release platform.

Liposomes have great tunability in physical/chemical properties for different therapeutic applications, however temporal and spatial controlled drug release from liposome carrier remain a challenge due to limited stability of conventional liposomes at physiologic conditions again drug leakage at non-target sites. In a recently study, a partially polymerized liposomal system was developed to improve the stability of liposome carrier as well as the precisely released capability. The partially polymerized liposomes (PPLs) were attached by gold nanoparticles $(50 \mathrm{~nm})$ on the surface as shown in Figure 8 and remain stable under physiological temperatures $\left(40^{\circ}\right)$ for three days, while releasing over $70 \%$ of the contents inside PPLs instantaneously after being triggered with a high energy laser light (Nd: YAG laser: $532 \mathrm{~nm}$ wavelength, $6 \mathrm{~nm}$ duration, $10 \mathrm{~Hz}$ repetition rate, energy $3.3 \mathrm{~W} / \mathrm{cm}^{2}$ ) matching the absorption peak of the GNP's. TEM images before and after high energy pulsed laser triggering demonstrated that the gold nanoparticles attached on the surface of PPLs were completely destroyed after being impinged by the high energy laser, which is most likely by the effect of melting and reforming into smaller particles.

By using the same laser but reduced energy, photoacoustic imaging can be used to evaluate the breakdown of gold nanoparticles and the drug release efficiency. As shown in Figure 9, two GNP solution targets are photoacoustic imaged. The controlled target is always filled with original GNP solution $(0.1 \mathrm{~mL})$ before high energy laser triggering.
The evaluating target is filled by original GNP solution $(0.1 \mathrm{~mL})$ before high energy and by damaged GNP solution $(0.1 \mathrm{~mL})$ after high energy triggering, in succession. From the image, we can immediately observe above $20 \%$ drop of the intensity on the photoacoustically reconstructed image. The GNP solution used in photoacoustic imaging is about $5 \times 10^{10}$ particles $/ \mathrm{mL}$, which is 20 -folder lower than the GNP solution in the reported study. If the higher concentrated GNP solution is used, the bigger difference in photoacoustic imaging is expected.

\section{Conclusion and Discussion}

In this paper, we provide a review of both the photoacoustic imaging technique and studies in diagnostic and theranostic applications. Photoacoustic imaging in breast cancer detection and osteoarthritis evaluation is included in the section of photoacoustic applications in diagnostic medicine, and a brief review of optical controlled drug release and photoacoustic evaluation is presented in the section of photoacoustic applications in theranostic medicine. Although the imaging capability of photoacoustic imaging seems not possible to compete with powerful MRI, which is capable of providing high quality images for different tissues by using large magnetic fields, the cost of photoacoustic imaging device is much lower. Photoacoustic imaging uses nonionizing visible/near infrared light and is very safe for patients, ideal for frequent-use situation such as in routine examination or preventive examination. Photoacoustic imaging has a scalable spatial resolution from several microns to millimeter, which may enable photoacoustic imaging a promising tool for broad applications in biomedicine. The photoacoustically resolved optical information (optical absorption, physiological/functional parameters such as hemoglobin level, oxygenation, water content) are highly sensitive biomarker to tumor/rheumatoid development and tissue metabolism. The availability of exogenous 
Citation: Sun Y, Jiang H, O’Neill BE (2011) Photoacoustic Imaging: An Emerging Optical Modality in Diagnostic and Theranostic Medicine. J Biosens Bioelectron 2:108. doi:10.4172/2155-6210.1000108

contrast agents and newly developed nanotechnology further extend the capability of photoacoustic imaging for early cancer detecting with imaging contrast enhancement, cells labeling/targeting, molecular imaging and evaluation of treatment response / drug release efficiency in theranostic studies. Overall, photoacoustic imaging is a promising tool, potential for both preclinical studies and clinical studies.

\section{References}

1. Bell AG (1880) On the production of sound by light. Am J Sci 20: 305.

2. Tam AC (1986) Application of photoacoustic sensing techniques. Rev Mod Phys 58: 381-431.

3. Oraevsky AA, Karabutov AA (2003) Optoacoustic tomography, Chapter 34 in Biomedical Photonics Handbook, T. Vo-Dinh, Ed., Vol. PM125, pp. 34/1-34/34 CRC Press, Boca Raton, FL.

4. Gu X, Zhang Q, Bartlett M, Schutz L, Fajardo L, et al. (2004) Differentiation of cysts from solid tumors in the breast with diffuse optical tomography. Acad Radiol 11: 53-60.

5. Hielscher AH, Klose AD, Scheel AK, Moa-Anderson B, Backhaus $M$, et al (2004) Sagittal laser optical tomography for imaging of rheumatoid finger joints. Phys Med Biol 49: 1147-1163.

6. Scheel AK, Backhaus M, Klose AD, Moa-Anderson B, Netz UJ, et al. (2005) First clinical evaluation of sagittal laser optical tomography for detection of synovitis in arthritic finger joints. Ann Rheum Dis 64: 239-245

7. Yuan Z, Zhang Q, Jiang $\mathrm{H}$ (2007) 3D diffuse optical tomography imaging of osteoarthritis: Initial results in finger joints. J Biomed Opt 12: 034001.

8. Duck FA (1990) Physical properties of tissue: a comprehensive reference book Academic Press, London, UK.

9. Xu M, Wang LV (2006) Photoacoustic imaging in biomedicine. Rev Sci Instrum 77: 041101-041122.

10. Brecht H, Su R, Fronheiser M, Ermilov SA, Conjusteau A, et al. (2009) Wholebody three-dimensional optoacoustic tomography system for small animals. J Biomed Opt 14: 064007.

11. Sun Y, Sobe ES, Jiang H (2011) First assessment of three-dimensional quantitative photoacoustic tomography for in vivo detection of osteoarthritis in the finger joints. Med Phys 38: 4009.

12. Wang X, Pang Y, Ku G, Xie X, Stoica G, et al. (2003) Noninvasive laser-induced photoacoustic tomography for structural and functional in vivo imaging of the brain. Nat Biotechnol 21: 803-806.

13. Laufer J, Zhang E, Raivich G, Beard P (2009) Three-dimensional noninvasive imaging of the vasculature in the mouse brain using a high resolution photoacoustic scanner. Appl Opt 48: D299-D306.

14. Wang X, Chamberland DL, Jamadar DA (2007) Noninvasive photoacoustic tomography of human peripheral joints toward diagnosis of inflammatory arthritis. Opt Lett 32: 3002-3004

15. Sun Y, Sobel E, Jiang H (2009) Quantitative three-dimensional photoacoustic tomography of the finger joints: an in vivo study. J Biomed Opt 14: 064002.

16. Wang X, Carson PL, Fowlkes JB, Bude RO, Jamadar DA, et al. (2006) Imaging of joints with laser-based photoacoustic tomography: An animal study. Med Phys 33: 2691

17. Chamberland DL, Agarwal A, Kotov N, Fowlkes JB, Carson PL, et al. (2008) Photoacoustic tomography of joints aided by an Etanercept-conjugated gold nanoparticle contrast agent-an ex vivo preliminary rat study. Nanotechnol 19: 095101

18. Savateeva EV, Karabutov AA, Motamedi M, Bell BA, Johnigan RM, et al. (2000) Noninvasive detection and staging of oral cancer in vivo with confocal optoacoustic tomography. Proc SPIE 3916: 55

19. Oraevsky AA, Karabutov AA, Solomatin SV (2001) Laser optoacoustic imaging of breast cancer in vivo. Proc SPIE 4526: 06-11.

20. Ermilov SA, Khamapirad T, Conjustea A, Leonar MH, Lacewell R, et al. (2009) Laser optoacoustic imaging system for detection of breast cancer. J Biomed Opt 14: 024007-1-14

21. Manohar S, Kharine A, van Hespen J CG, Steenbergen W, van Leeuwen TJ
(2004) Photoacoustic mammography laboratory prototype: imaging of breast tissue phantoms. J Biomed Opt 9: 1172. http://spiedigitallibrary.org/jbo/resource/1/jbopfo/v9/i6/p1172 s1?isAuthorized=no

22. Manohar S, Vaartjes SS, van Hespen JCG, Klaase JM, van den Engh FM, et al. (2007) Initial results of in vivo non-invasive cancer imaging in the human breas using near-infrared photoacoustics. Opt Exp 15: 12277-12285.

23. Zhang HF, Maslov K, Stoica G, Wang LV (2006) Functional photoacoustic microscopy for high-resolution and noninvasive in vivo imaging. Nat Biotechno 24: 848-851.

24. Zhang HF, Maslov K, Stoica G, Wang LV (2007) In vivo imaging of subcutaneous structures using functional photoacoustic microscopy. Nat Protoc 2 797-804.

25. Viator JA, Au G, Paltauf G, Jacques S, Prahl S, (2002) Clinical testing of a photoacoustic probe for port wine stain depth determination. Lasers Surg Med 30: 141-148.

26. Kim C, Song KH, Gao F, Wang LV (2010) Sentinel Lymph Nodes and Lymphat ic Vessels: Noninvasive Dual-Modality in Vivo Mapping by Using Indocyanine Green in Rats-Volumetric Spectroscopic Photoacoustic Imaging and Planar Fluorescence Imaging. Radiol 255: 442-450.

27. Kim C, Song H, Cai X, Yao J, Wei A (2011) In vivo photoacoustic mapping of lymphatic systems with plasmon-resonant nanostars. J Mat Chem 21: 2841 2844

28. Zhang Q, Liu Z, Carney PR, Yuan Z, Chen H, et al. (2008) Non-invasive imag ing of epileptic seizures in vivo using photoacoustic tomography. Phys Med Biol 53: 1921-1931.

29. Aguirre A, Guo P, Gamelin J, Yan S, Sanders MM, et al. (2009) Coregistered three-dimensional ultrasound and photoacoustic imaging system for ovarian tissue characterization. J Biomed Opt 14: 054014

30. Agarwal A, Huang SW, O’Donnell M, Day KC, Day M, et al. (2007) Targeted gold nanorod contrast agent for prostate cancer detection by photoacoustic imaging. J Appl Phys 102: 064701.

31. Zerda A, Paulus YM, Teed R, Bodapati S, Dollberg Y, et al. (2010) Photoacoustic ocular imaging. Opt Lett 35: 270-272.

32. Silverman RH, Kong F, Chen YC, Lloyd HC, Kim HH, et al. (2010) High-Resolution Photoacoustic Imaging of Ocular Tissues. Ultrasound Med Biol 35: 733

33. Emelianov SY, Li P, O’Donnell M (2009) Photoacoustics for molecular imaging and therapy. Phys Today 62: 34-39.

34. Razansky D, Distel M, Vinegoni C, Ma R, Perrimon N, et al. (2009) Multispectral opto-acoustic tomography of deep-seated fluorescent proteins in vivo Nat. Photonics 3, 379-379.

35. Brecht HP, Su R, Fronheiser M, Ermilov SA, Conjusteau A (2009) Wholebody three-dimensional optoacoustic tomography system for small animals. Biomed Opt 14: 064007-1-8.

36. Zerda ADL, Zavaleta1 C, Keren S, et al. (2008) Carbon nanotubes as photoacoustic molecular imaging agents in living mice. Nat Nanotechnol 3: 557-562.

37. Kim J, Galanzha El, Shashkov EV, Moon Golden H, Zharov VP (2009)GoldCarbon nanotubes as multimodal photoacoustic and photothermal high-contrast molecular agents. Nat Nanotechnol 4: 688-694.

38. Galanzha El, Shashkov EV, Kelly T, Kim J, Yang T, et al. (2009) In vivo magnetic enrichment and multiplex photoacoustic detection of circulating tumou cells. Nat Nanotechnol 4: 855-860.

39. Ntziachristos V, Razansky D (2010) Molecular Imaging by Means of Multispectral Optoacoustic Tomography (MSOT). Chem Rev 110: 2783-2794.

40. Razansky D, Buehler A, Ntziachristos V (2011) Volumetric real-time multispectral optoacoustic tomography of biomarkers. Nat Protocols 6: 1121-1129.

41. Boucharda LS, Anwarb MS, Liu G, Hann B, Harry Xie Z, et al. (2009) Picomola sensitivity MRI and photoacoustic imaging of cobalt nanoparticles. PNAS 106 4085-4089.

42. Sun $Y$, Jiang $H$ (2009) Quantitative three-dimensional photoacoustic tomography of the finger joints: phantom studies in a spherical scanning geometry. Phys Med Biol 54: 5457-5467. 
Citation: Sun Y, Jiang H, O’Neill BE (2011) Photoacoustic Imaging: An Emerging Optical Modality in Diagnostic and Theranostic Medicine. J Biosens Bioelectron 2:108. doi:10.4172/2155-6210.1000108

43. Xi L, Sun J, Zhu Y, Wu L, Xie H, et al. (2010) Photoacoustic imaging based on MEMS mirror scanning. Biomed. Opt Express 1: 1278-1283.

44. Yang JM, Maslov K, Yang HC, Zhou QF, Shung KK, et al. (2009) Photoacoustic endoscopy. Opt Lett 34: 1591-1593.

45. Yuan Y, Yang S, Xing D (2010) Preclinical photoacoustic imaging endoscope based on acousto-optic coaxial system using ring transducer array. Opt Lett 35: 2266-2268

46. Karpiouk AB, Wang B, Emelianov SY (2010) Development of a catheter for combined intravascular ultrasound and photoacoustic imaging. Rev Sci Instrum 81: 014901.

47. Yao DK, Maslov K, Shung KK, Zhou QF, Wang LV (2010) In vivo label-free photoacoustic microscopy of cell nuclei by excitation of DNA and RNA. Opt Lett 35: 4139-4141.

48. Maslov K, Zhang HF, Hu S, Wang LV (2008) Optical-resolution photoacoustic microscopy for in vivo imaging of single capillaries. Opt Lett 33: 929-931.

49. Hu S, Maslov K, Wang LV (2011) Second-generation optical-resolution photoacoustic microscopy with improved sensitivity and speed. Opt Lett 36: 1134 1136.

50. Allen TJ, Cox BT, Beard PC (2005) Generating photoacoustic signals using high-peak power pulsed laser diodes. SPIE 5697: 233-242.

51. Kolkman RGM, Steenbergen W (2006) In vivo photoacoustic imaging of blood vessels with a pulsed laser diode. Lasers Med Sci 21: 134-139.

52. M. Xu, L. V. Wang (2003) Analytic explanation of spatial resolution related to bandwidth and detector aperture size in thermoacoustic or photoacoustic reconstruction. Phys Rev E 67: 056605

53. Yin B, Xing D, Wang Y, Zeng $Y$, Tan $Y$, et al. (2004) Fast photoacoustic imaging system based on 320-element linear transducer array. Phys Med Biol 49 1339-1346.

54. Yang D, Xing D, Gu H, Tan Y, Zeng L (2005) Fast multielement phase-controlled photoacoustic imaging based on limited-field-filtered back-projection algorithm. Appl Phys Lett 87: 194101-1941011-3.

55. Xu Y, Wang LV, Ambartsoumian G, Kuchment $P$ (2004) Reconstructions in limited-view thermoacoustic tomography. Medical Physics 31: 724-733.

56. Andreev VG, Karabutov AA, Solomatin SV, Savateeva EV, Aleinikov V, et al (2000) Optoacoustic tomography of breast cancer with arc-array transducer. Proc SPIE 3916: 36.

57. Gamelin J, Aguirre A, Maurudis A, Huang F, Castillo D, et al. (2008) Curved array photoacoustic tomographic system for small animal imaging. Journal of Biomedical Optics 13: 024007 (1-10).

58. Gamelin J, Maurudis A, Aguirre A, Huang F, Guo P, et al. (2009) A real-time photoacoustic tomography system for small animals. Optics Express 17: 1048910498

59. Zhang EZ, Beard P (2004) Ultra high sensitivity, wideband Fabry Perot ultrasound sensors as an alternative to piezoelectric PVDF transducers for biomedical photoacoustic detection. Proc SPIE 5320: 222-229.

60. Köstli KP, Beard PC (2003) Two-Dimensional Photoacoustic Imaging by Use of Fourier-Transform Image Reconstruction and a Detector with an Anisotropic Response. Appl Opt 42: 1899-1908.

61. Beard PC, Zhang EZ, Cox BT (2004) Transparent Fabry Perot polymer film ultrasound array for backward-mode photoacoustic imaging. Proc SPIE 5320: 230-237.

62. Ashkenazi A, Hou Y, Buma T, O’Donnell M (2005) Optoacoustic imaging using thin polymer etalon. Appl Phys Lett 86: 134102.

63. Niederhauser JJ, Jaeger M, Lemor R, Weber P, Frenz M (2005) Combined ultrasound and optoacoustic system for real-time high-contrast vascular imaging in vivo. IEEE Transaction on Medical Imaging 24: 436-440.

64. Kostli KP, Frenz M, Bebie H, Weber HP (2001) Temporal backward projection of optoacoustic pressure transients using Fourier transform methods. Phys Med Biol 46: 1863-1872.

65. Hoelen CGA, de Mul FF, Pongers R, Dekker A (1998) Three dimensional photoacoustic imaging of blood vessels in tissue. Opt Lett 23: 648-650.

66. Liu PY (1998) The P-transform and photo acoustic image reconstruction. Phys Med Biol 43: 667-674.
67. Zhulina YV (2000) Optical statistical approach to optoacoustic image reconstruction. Appl Opt 39: 5971-5977.

68. Kostli KP, Frauchiger D, Niederhauser J, Paltauf G, Weber H (2001) Optoacoustic imaging using a three-dimensional reconstruction algorithm. IEEE J Sel Top Quantum Electron 7: 918-923.

69. Jiang H, Yuan Z, Gu X (2006) Spatially varying optical and acoustic property reconstruction using finite-element-based photo acoustic tomography. $\mathrm{J}$ Op Soc Am A 23: 878-888.

70. Yuan Z, Zhang Q, Jiang H (2006) Simultaneous reconstruction of acoustic and optical properties of heterogeneous media by quantitative photo acoustic tomography. Opt Exp 14: 6749-6754.

71. Yuan Z, Jiang H (2007) Three-dimensional finite-element-based photo acoustic tomography: Reconstruction algorithm and simulations. Med Phys 34: 538546 .

72. Xu Y, Wang LV (2003) Effects of acoustic heterogeneity on thermoacoustic tomography in the breast. IEEE Transactions on Ultrasonics Ferroelectrics and Frequency Control 50: 1134-1146.

73. Ripoll J, Ntziachristos V (2005) Quantitative point source photo acoustic inversion formulas for scattering and absorbing medium. Phys Rev E Nonlin Sof Matter Phys 71: 031912.

74. Yuan Z, Jiang H (2006) Quantitative photoacoustic tomography: recovery of optical absorption coefficient map of heterogeneous medium. Appl Phys Let 88: 231101.

75. Laufer J, Delpy D, Elwell C, Beard P (2007) Quantitative spatially resolved measurement of tissue chromophore concentrations using photoacoustic spectroscopy: application to the measurement of blood oxygenation and haemoglobin concentration. Phys Med Biol 52: 141-168.

76. Yuan Z, Jiang H (2009) Simultaneous recovery of tissue physiological and acoustic properties and the criteria for heterogeneous media by quantitative photo acoustic tomography. Opt Lett 34: 1714-1716.

77. Srinivasan S, Pogue BW, Jiang SD, Dehghani H, Kogel C, et al. (2003) Interpreting hemoglobin and water concentration, oxygen saturation, and scattering measured in vivo by near-infrared breast tomography. Proc Natl Acad Sci USA $100,12349-12354$

78. Yuan Z, Jiang H (2009) Quantitative photo acoustic tomography. Philos Transact A Math Phys Eng Sci 367: 3043-3054 (2009).

79. Yuan Z, Zhang Q, Sobel ES, Jiang H (2008) Tomographic x-ray-guided threedimensional diffuse optical tomography of osteoarthritis in the finger joints. $J$ Biomed Opt 13: 044006

80. Siphanto RI, Thumma KK, Kolkman RGM, van Leeuwen TG, de mul FFM, et al. (2005) Serial noninvasive photoacoustic imaging of neovascularization in tumor angiogenesis. Opt Express 13: 89-95.

81. Doyle AJ, Murray KA, Nelson EW (1995) Selective use of image-guided largecore needle biopsy of the breast: accuracy and cost-effectiveness. Am J Roentgenol 165: 281-284

82. Liberman L, Feng TL, Dershaw DD (1998) US-guided core breast biopsy: use and cost-effectiveness. Radiol 208: 717-723.

83. Rosen EL, Sickles EA, Keatin D (1999) Ability of mammography to reveal nonpalpable breast cancerin women with palpable breast masses. Am J Roentgenol 172: 309-312.

84. Jiang H, Iftimia NV, Xu Y, Eggert JA, Fajardo LL, et al. (2002) Near-Infrared Optical Imaging of the Breast with Model-Based Reconstruction. Acad Radiol 9: 186-194.

85. Yang S, Xing D, Zhou Q, Xiang L, Lao Y (2007) Functional imaging of cerebrovascular activities in small animals using high-resolution photoacoustic tomography. Med Phys 34: 3294-3301.

86. Oddi CV (1996) New Perspective on Osteoarthritis. Am J Med 100: 10S-15S.

87. Moskowitz RW, Altman RD, Hochberg MC, Buchwalter JA, Goldberg VM (2007) Osteoarthritis: diagnose and medical/surgical management. Lippincott Williams \& Wilkins. JAMA 299: 1840-1841.

88. Sarzi-Puttini P, Cimmino MA, Scarpa R, Caporali R, Parazzini F, et al. (2005) Osteoarthritis: an overview of the disease and its treatment strategies. Semin Arthritis Rheum 35: 1-10. 
Citation: Sun Y, Jiang H, O'Neill BE (2011) Photoacoustic Imaging: An Emerging Optical Modality in Diagnostic and Theranostic Medicine. J Biosens Bioelectron 2:108. doi:10.4172/2155-6210.1000108

89. Abramson SB, Attur M, Yazici Y (2006) Prospect for disease modification in osteoarthritis. Nat Clin Pract Rheumatol 2: 304-312.

90. Meisel AD, Bullough PG (1984) Atlas of Osteoarthritis. Gower Medical Publishing, New York.

91. Beuthan J, Prapavat V, Naber R, Minet O, Muller G (1996) Diagnostic of inflammatory rheumatic diseases with photon density waves. Proc SPIE 2676: 43-53.

92. Prapavat V, Runge W, Mans J, Krause A, Beuthan J, et al. (1997) The development of a finger joint phantom for the optical simulation of early inflammatory rheumatic changes. Biomed Tech 42: 319-326.

93. Yuan Z, Zhang Q, Sobel E, Jiang H (2007) Three-dimensional diffuse optical tomography of osteoarthritis: initial results in the finger joints. J Biomed Opt 12: 034001.

94. Yuan Z, Zhang Q, Sobel ES, Jiang H (2008) Tomographic x-ray guided threedimensional diffuse optical tomography of osteoarthritis in the finger joints. $J$ Biomed Opt 13: 044006.

95. Hielscher AH, Klose AD, Scheel AK, Moa-Anderson B, Backhaus $M$ et al. (2004) Sagittal laser optical tomography for imaging of rheumatoid finger joints. Phys Med Biol 49: 1147-1163.

96. Scheel AK, Backhaus M, Klose A, Moa-Anderson B, Netz U, et al. (2005) First clinical evaluation of sagittal laser optical tomography for detection of synovitis in arthritic finger joints. Ann Rheum Dis 64: 239-245.

97. Yuan Z, Zhang Q, Jiang H (2006) Simultaneous reconstruction of acoustic and optical properties of heterogeneous media by quantitative photoacoustic tomography. Opt Express 14: 6749-6754.
98. Jin X, Wang LV (2006) Thermoacoustic tomography with correction for acoustic speed variations. Physics in Medicine and Biology 51: 6437-6448.

99. Jin X, Li CH, Wang LV (2008) Effects of acoustic heterogeneities on transcranial brain imaging with microwave-induced thermoacoustic tomography. Med Phys 35: 3205-3214.

100. Hahn MA, Singh AK, Sharma P, Brown SC, Moudgil BM (2011) Nanoparticles as contrast agents for in-vivo bioimaging: current status and future perspectives. Anal Bioanal Chem 399: 3-27.

101. Janib SM, Moses AS, MacKay JA (2010) Imaging and drug delivery using theranostic nanoparticles. Adv Drug Deliv Rev 62: 1052-1063.

102. Xie J, Lee S, Chen X (2010) Nanoparticle-based theranostic agents. Adv Drug Deliv Rev 62: 1064-1079.

103. McCarthy JR (2010) Multifunctional agents for concurrent imaging and therapy in cardiovascular disease. Adv Drug Deliv Rev 62: 1023-1030.

104. Pan D, Lanza GM, Wickline SA, Caruther SD (2009) Nanomedicine: perspective and promises with ligand-directed molecular imaging. Eur J Radiol 70 274-285

105. Rapoport N (2007) Physical stimuli-responsive polymeric micelles for anticancer drug delivery. Prog Polym Sci 32: 962-990.

106. MacEwan S, Callahan D, Chilkoty A (2010) Stimulus-responsive macromolecules and nanoparticles for cancer drug delivery. Nanomed 5: 793-806.

107. Qin G, Li Z, Xia R, Li F, O'Neill BE, et al. (2011) Partially polymerized liposomes: stable against leakage yet capable of instantaneous release for remote controlled drug delivery. Nanotechnol 22, 155605 